Revue des patrimoines

Le patrimoine religieux des $\mathrm{XIX}^{\mathrm{e}}$ et $\mathrm{XX} \mathrm{X}^{\mathrm{e}}$ siècles

\title{
La chapelle de l'ancien hôpital Saint-Lazare (Paris)
}

\section{Agnès Chauvin}

\section{OpenEdition}

\section{Journals}

Édition électronique

URL : http://journals.openedition.org/insitu/4578

DOI : $10.4000 /$ insitu.4578

ISSN : 1630-7305

\section{Éditeur}

Ministère de la culture

\section{Référence électronique}

Agnès Chauvin, « La chapelle de l'ancien hôpital Saint-Lazare (Paris) », In Situ [En ligne], 11 | 2009, mis en ligne le 18 avril 2012, consulté le 30 avril 2019. URL : http://journals.openedition.org/insitu/4578 ; DOI : $10.4000 /$ insitu. 4578

Ce document a été généré automatiquement le 30 avril 2019

\section{(c) (i) $\odot$}

In Situ Revues des patrimoines est mis à disposition selon les termes de la licence Creative Commons Attribution - Pas d'Utilisation Commerciale - Pas de Modification 4.0 International. 


\title{
La chapelle de l'ancien hôpital Saint-Lazare (Paris)
}

\author{
Agnès Chauvin
}

\section{Le quadrilatère Saint-Lazare au fil du temps}

$1 \mathrm{Au} \mathrm{XII}$ siècle, une léproserie, fondée par les hospitaliers Saint-Lazare de Jérusalem, occupe le terrain formé par les actuels boulevard de la Chapelle, rue du Faubourg SaintDenis, rue de Paradis et rue du Faubourg Poissonnière ${ }^{1}$. Cette léproserie bénéficie de la protection royale et accueille un grand nombre de malades jusqu'au dernier cas de lèpre signalé en 1597.

Les lazaristes prennent possession des bâtiments en 1632 et les agrandissent, entre 1681 et 1685 , selon les multiples vocations de la congrégation: secours aux malades, aux galériens et aux esclaves, séminaires et maison de correction pour jeunes gens dissipés ou libertins dont Beaumarchais fut l'un des plus illustres ${ }^{23}$.

Dès juillet 1789, la maison est pillée puis la congrégation des lazaristes supprimée et un décret de la Convention reconnait Saint-Lazare comme prison officielle en 1794. Les bâtiments sont alors administrés par la préfecture de police. Simples prévenues, criminelles lourdement condamnées et prostituées en provenance des prisons de la Force, Vincennes et de la Salpêtrière se trouvent réunies à Saint-Lazare. La prison est décrite comme un des sites les plus sinistres et les plus pathétiques de Paris. Les bâtiments sont cédés au département de la Seine.

4 Un des quartiers de la prison, celui de "La Pistole", est assez bien connu. Dans cette section, les détenues sont traitées avec certains égards. Parmi les " pistolières » célèbres, on retrouve les noms de Louise Michel et de Mme Caillaux. Plus tard, des espionnes, comme Mata Hari, seront incarcérées à Saint-Lazare.

5 À partir de 1838, à l'initiative du baron Delessert, préfet de police, la garde des détenues par des femmes est enfin acquise ${ }^{4}$. Celles de Saint-Lazare sont alors placées sous la 
direction des Sœurs de Marie-Joseph pour les Prisons de 1850 à 1927, date de la démolition de la prison ${ }^{5}$.

6 Après cette date, les détenues de Saint-Lazare sont réparties dans les différentes prisons parisiennes. L'ancienne prison transformée par les constructions des années 1930 devient «maison de santé Saint-Lazare » et l'infirmerie est reconvertie en unité de vénérologie. Elle ne fermera qu'en 1955.

7 En 1961, la préfecture de police cesse d'administrer cet hôpital et cède l'infirmerie à l'Assistance publique. Après sa désaffectation, la chapelle sert d'amphithéâtre à la faculté de médecine de Paris et l'infirmerie accueille un petit service de gastro-entérologie qui rejoint définitivement celui de l'hôpital Lariboisière en 1988. L'A.P.-H.P.remet alors la totalité des bâtiments de l'ancien hôpital à la Ville de Paris.

8 Tout un ensemble d'équipements culturels et sportifs, d'enseignement et de centre social de quartier doit ouvrir prochainement dans les anciens bâtiments rénovés par l'agence Atelier Canal.

\section{La réorganisation de la prison et les constructions de Pierre-Louis Baltard}

9 L'église du XII ${ }^{e}$ siècle, qui avait reçu de multiples affectations depuis la Révolution grenier à foin, magasin à farine - ne cessait de se dégrader et certains bâtiments menaçaient ruine. L'église est démolie en 1823 et l'administration pénitentiaire entreprend de réorganiser l'ensemble des bâtiments ${ }^{6}$. Elle décide la construction d'une chapelle et d'une infirmerie sur l'emplacement des jardins situés à l'arrière des bâtiments du XVII siècle ${ }^{7}$. (fig. $\mathbf{n}^{\circ} \mathbf{1}$ ) 


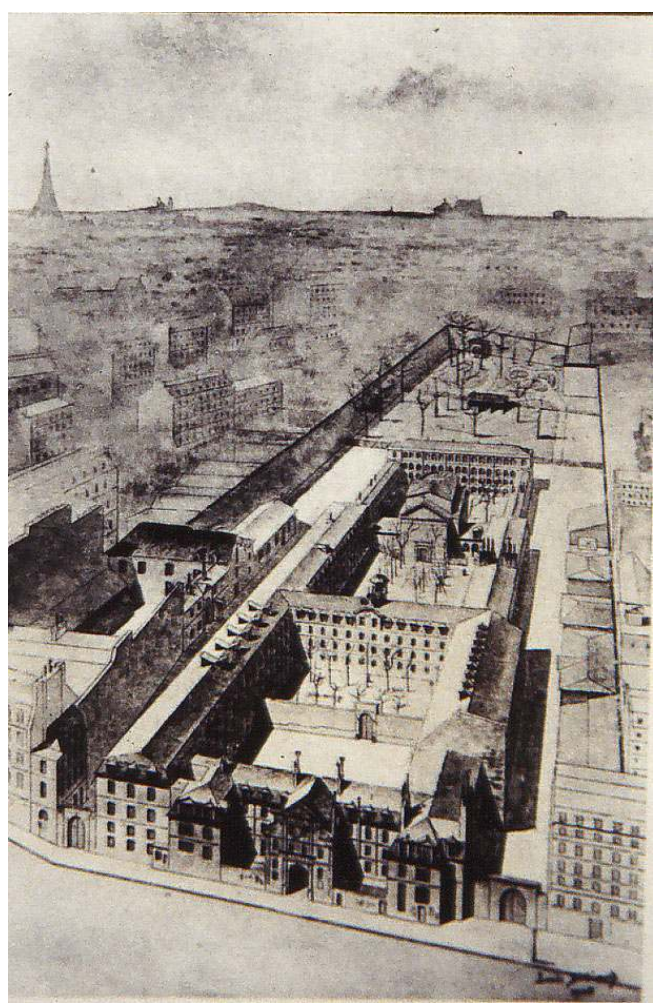

Vue de la prison Saint-Lazare, fin XIXe siècle, archives de l'A.P.-H.P. [cote HZ 107]

Phot. Claire Vignes-Dumas, 2004. (c) CRMH Île-de-France

La construction de la nouvelle chapelle et de l'infirmerie, située à l'arrière du chevet, est confiée en 1824 à Louis-Pierre Baltard (1764-1846) par le comte de Chabrol, préfet de la Seine. L'infirmerie, appelée Infirmerie spéciale est construite pour améliorer les conditions de certaines détenues. Elle soigne celles de Saint-Lazare ainsi que les malades internées dans les prisons de Broca et de La Pitié. Professeur de théorie à l'École des Beaux-Arts, Louis-Pierre Baltard est l'architecte des prisons de Paris depuis $1813^{8}$. Il a déjà effectué des travaux d'aménagement dans les prisons parisiennes de La Force et des Madelonnettes (1817-1818) et construit la prison de Draguignan en 1820. Parallèlement à la chapelle Saint-Lazare, il construit la chapelle de la prison Sainte-Pélagie (1764-1846) ${ }^{9}{ }^{10}$. Devenu en 1825 l'architecte des prisons et des tribunaux de la Seine, il construira le palais de justice de Lyon (1835-1846) ${ }^{11}$. Préoccupé par les conditions d'enfermement des détenus et à la recherche d'une architecture la plus adaptée à leur situation, Pierre-Louis Baltard a publié en 1829 l'Architectonographie des prisons. Cette œuvre théorique et pratique s'inscrit dans les décennies majeures de l'histoire de l'architecture carcérale, au moment des premières réflexions et des réalisations les plus nombreuses ${ }^{12}$. 


\section{La chapelle}

\section{Le plan}

La chapelle constitue le centre de l'ensemble carcéral et Pierre-Louis Baltard recommande de la placer au centre de l'établissement, dans l'axe majeur, comme le bâtiment de l'administration situé à l'entrée ${ }^{13}$.

Figure 2

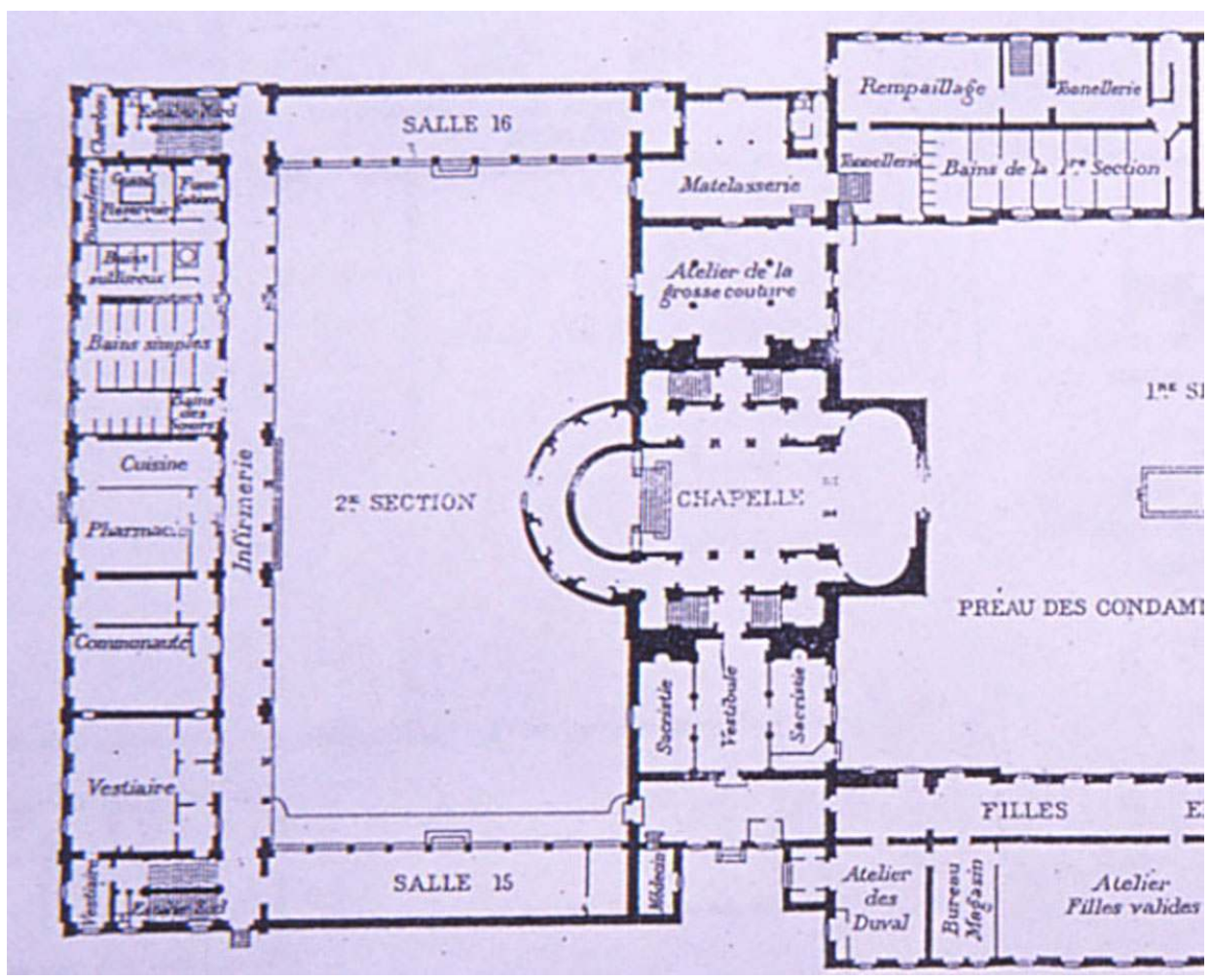

Plan de la prison en 1912 publié dans Commission du Vieux-Paris, 1912

Phot. Agnès Chauvin, 2002. (c) CRMH île-de-France

12 La chapelle cruciforme a adopté un plan pseudo-basilical, comme la plupart des églises construites dans les années 1830 , bien que ce plan ait fait l'objet de nombreuses critiques. Le plan de la chapelle est à considérer depuis les galeries plutôt que depuis le sol de la nef. La nef apparait comme une sorte de sous-sol ouvert ou comme une fosse et les galeries latérales étonnent par leur ampleur. Elles permettaient aux détenues des accès multiples à l'église puisque les différentes catégories de condamnées devaient être séparées dans la nef. Aujourd'hui ne subsiste qu'une entrée latérale, alors que les plans de Baltard permettent d'en distinguer trois : l'entrée principale et deux entrées latérales de chaque côté du transept ${ }^{14}$. (fig. $\mathbf{n}^{\circ}$ 2)

\section{La façade}

La façade de la chapelle de Baltard, en pierre, ne comportait aucun décor ni aucune ouverture à l'exception de la porte d'entrée. Donnant sur une cour de promenade, elle 
offrait un aspect austère que la façade actuelle, rhabillée de briques mécaniques, ornée d'un blason et percée d'un oculus par Gaston Lefol a fait oublier ${ }^{15}$. (fig. $\mathbf{n}^{\circ}$ 3)

Figure 3

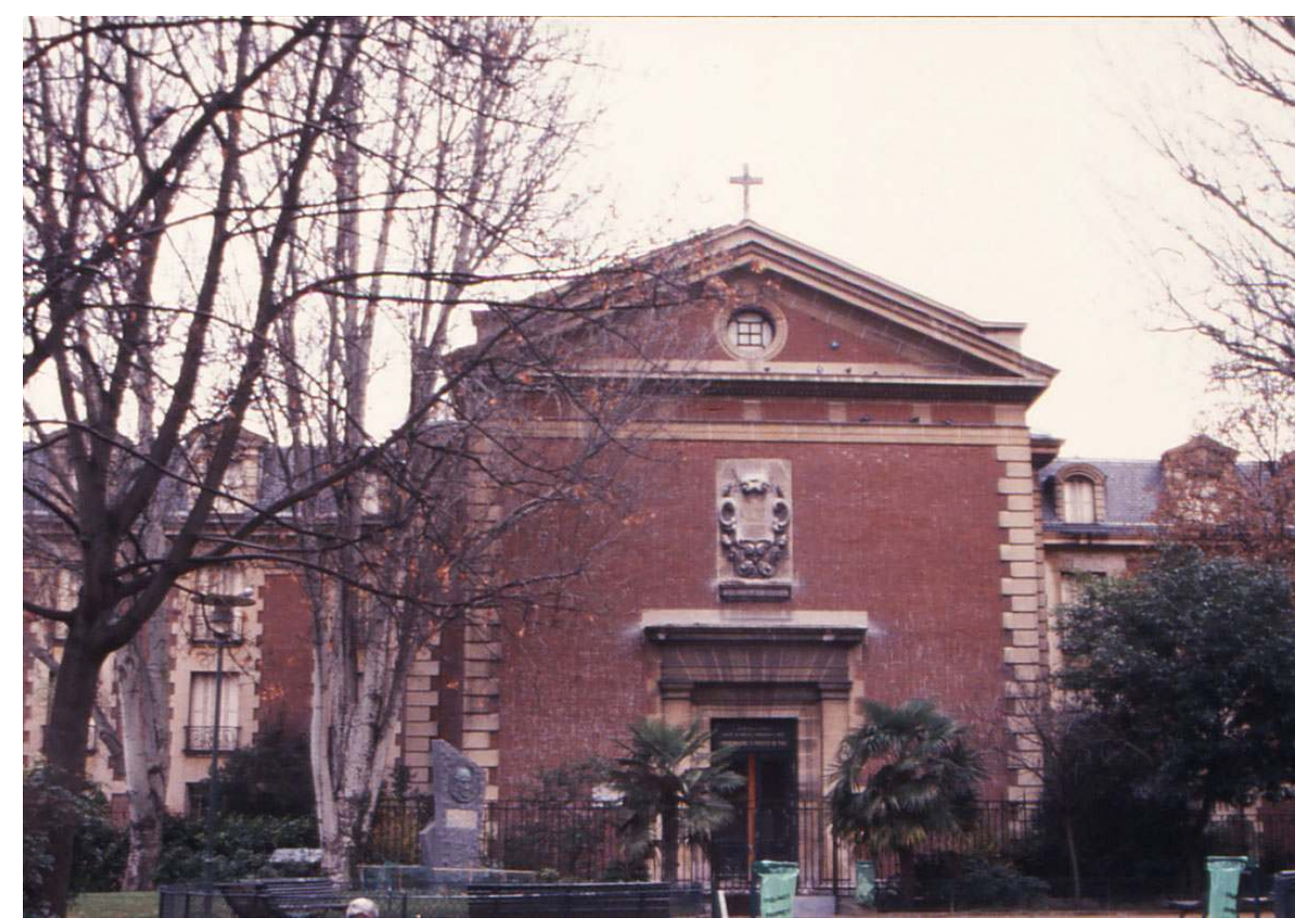

Façade de l'ancienne chapelle

Phot. Claire Vignes-Dumas, 2004. (c) CRMH Île-de-France

\section{Le chevet}

De simples pilastres doriques rythment cette façade en alternance avec les six verrières de l'abside. Au-dessus, l'entablement souligne la justesse des proportions d'origine aujourd'hui masquée par une surélévation contemporaine des travaux de Lefol ${ }^{16}$.

\section{La nef}

15 À l'intérieur, l'autel est dans une position dominante, à l'étage, au-dessus de toute hiérarchie humaine. "Des multiples images d'autorité offertes au prisonnier, celle de l'aumônier, principal agent des améliorations morales, est naturellement la plus riche ${ }^{17}$. L'abside est occupée par trois volées successives de marches qui permettaient cette progression vers l'autel. Elle est éclairée par six verrières qui sont les seules ouvertures de la chapelle. Des escaliers de part et d'autre de l'abside descendent vers deux pièces qui ont dû servir de chapelles secondaires ${ }^{18}$.

De fortes colonnes aux chapiteaux et bases doriques supportent un puissant entablement. Fortement imprégné de culture antique, Louis-Pierre Baltard affirme une fidélité passionnée aux monuments romains. La nef est couverte d'une voûte en berceau et l'abside voûtée en cul-de-four conserve un décor de faux caissons ${ }^{19}$. Il n'y a aucun autre décor sur les frises ou les chapiteaux ${ }^{20}$. (fig. $\mathbf{n}^{\circ} 4$ ) 
Figure 4

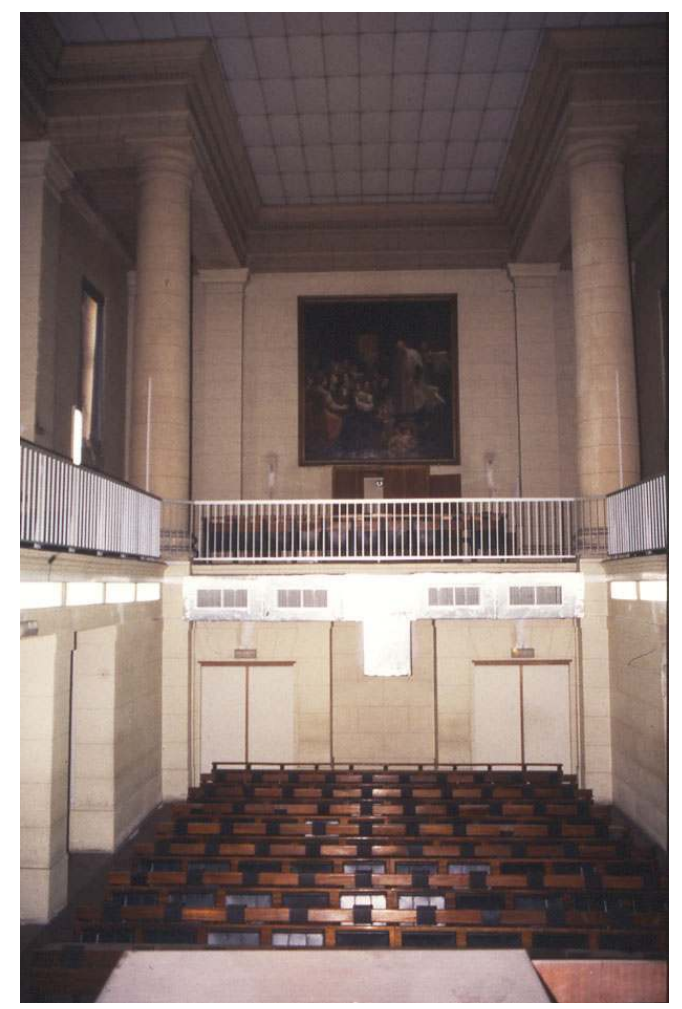

Vue de la nef de l'ancienne chapelle

Phot. Agnès Chauvin, 2002. (C) CRMH Île-de-France

\section{De la prison à l'hôpital}

La démolition de la prison Saint-Lazare, symbole d'horreur et de déchéance, est régulièrement envisagée dès la fin du XIX siècle. Plusieurs dizaines d'années d'études, perturbées par la guerre et des revirements ralentissent le projet d'humanisation des bâtiments de la prison ${ }^{21}$.

En 1928, le Conseil général de la Seine décide de démolir les bâtiments de la maison d'arrêt et de correction. Il estime qu'il réalise là «une opération qui restera à son honneur $»^{22}$. Mais un peu plus tard, la Commission du Vieux-Paris demande «la conservation du guichet d'entrée, de la porte d'entrée du réfectoire, de la rampe d'escalier en fer forgé du bâtiment de l'administration, de la clochette d'argent et du fronton de la façade qui, si son état en permet le démontage, pourrait être utilisé dans les

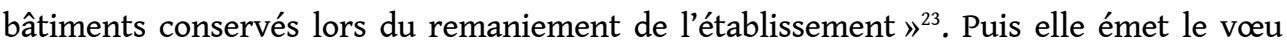
que tous les éléments antérieurs au XIXe siècle soient classés au titre des monuments historiques ${ }^{24}$. Lors de sa séance du 21 juin 1930, la Commission supérieure des monuments historiques ne propose que le classement du pavillon d'entrée et il est souligné que la suppression de la maison d'arrêt est à l'étude depuis 1906. Mais Paul Léon, sous-secrétaire d'État aux Beaux-Arts écrit, en mars 1931, que «le déplacement de cette façade lui enlèverait son intérêt historique et que l'opération ne justifiait pas la dépense (...). Il a donc lieu de renoncer à tout classement de la prison Saint-Lazare malgré les souvenirs 
qui s'y rattachaient ${ }^{25}$. En 1933, de nombreux articles s'émeuvent de la destruction des anciens bâtiments de l'hôpital ${ }^{26}$.

\section{Les travaux de Gaston Lefol}

19

En mars 1931, le projet définitif de Gaston Lefol (1874- ?) est adopté. Il comprend la conservation et la remise en état de la chapelle et de l'infirmerie de Louis-Pierre Baltard ainsi que la construction de bâtiments de part et d'autre de la chapelle. Ces deux bâtiments sont construits à la place des bâtiments existants qui doivent être rasés ${ }^{27}$. Ils comportent deux étages sur rez-de-chaussée et sous-sol. Ils étaient réservés, au nord, au personnel et au sud, à la communauté religieuse. Le bâtiment du personnel abritait le bureau du directeur de la prison, la salle de greffe, la salle de fouille; le bâtiment des religieuses comprenait des logements, un réfectoire, une cuisine, une lingerie et une sacristie.

La chapelle de l'ancien hôpital Saint-Lazare est une œuvre assez peu connue de LouisPierre Baltard dont on sait surtout qu'il a écrit L'Architectonographie des prisons et construit le palais de justice de Lyon, jalon dans l'histoire de l'architecture néoclassique. La nouvelle affectation de l'hôpital permettra d'apprécier les théories que Baltard a pu réaliser dans cet ancien bien national aménagé en prison.

1 Les nouveaux aménagements et l'ouverture au public de cet ensemble donneront aussi l'occasion de mieux connaître l'histoire de ce lieu un peu oublié de Paris.

Considérant que les bâtiments subsistants de l'ancienne prison Saint-Lazare constituent un des rares exemples parisiens d'architecture néoclassique construits par Louis-Pierre Baltard, l'ensemble des bâtiments, les façades et toitures de l'ancienne infirmerie, le sol de la cour et la totalité de la chapelle sont inscrits au titre des monuments historiques depuis le 28 novembre $2005^{28}$.

BIBLIOGRAPHIE

\section{Iconographie}

\section{Archives de l'assistance publique}

Photos cote 109 1B, 2B, 3B, 4B, 5B, 6B, 7B; 109. 26, 29, 30, 31 ; HZ 090, 094,107, 144 ; B5239-73nk et B5063-73 bis

\section{Bibliothèque nationale, cabinet des estampes}

E1-13 (165) : photographie de la façade de la prison par l'agence Rol

E0 109b boite 21* : photographie de la façade de la prison par Eugène Atget 
Va 289, t. 7 (cote mc H 072854-072974) : prison Saint-Lazare

\section{Musée Carnavalet, cabinet des arts graphiques}

$146^{\mathrm{E}}$ Topo : l'hôpital avant sa démolition, la chapelle actuelle

XXXVIII C : vues intérieures de l'ancien hôpital

Photothèque de la parisienne de photographie

www.roger-viollet.fr

\section{Sources}

\section{Archives de l'assistance publique-hôpitaux de Paris}

Plans G. Lefol 793 Foss 54-2/1,2,3,4

\section{Archives départementales}

Fonds préfectoral concernant l'entretien des bâtiments :

D7N4 39 (1873-1894)

D7N4 40 (1897-1900)

D7N4 41 (1921-1933)

4AZ 1154 ; maisons centrales, prison Saint-Lazare, démolition de l'église

$1326 \mathrm{~W}$ art.38 : évacuation de la prison en 1925

\section{Archives nationales}

Fonds Baltard $332 \mathrm{AP}, 1,20$ et 22

$\mathrm{F}^{19} 1902$, pièces 3209-3210(calques microfilmés)

\section{Bibliothèque historique de la Ville de Paris}

Procès-verbaux de la Commission du Vieux-Paris : 9 juillet 1912, 28 janvier, 25 février 1928 ; 29 novembre 1930, 9 mai 1931 ; 28 janvier, 25 février, $1^{\mathrm{er}}$ avril, 24 juin 1933.

\section{Médiathèque du patrimoine}

0081/075610/008 : courriers relatifs à la protection et à la démolition de l'ancienne prison (1884-1885 puis 1931-1933) 


\section{Bibliographie}

Beaumont-Maillet, Laure. Vie et histoire du Xe arr. Paris : Hervas, 1988.

Blizard, Léon et Campon, Jane. La prison Saint-Lazare : des origines à nos jours. Paris : 1911

(cote B.N Est. Ve 1043-4).

Pinon, Pierre. Louis-Pierre et Victor Baltard. Paris : Monum, Éditions du Patrimoine, 2005.

\section{NOTES}

1. Je remercie Claire Vignes-Dumas, documentaliste-recenseur à la C.R.M.H. qui m'a fait bénéficier de ses recherches effectuées pour la constitution du dossier de recensement.

2. Saint Vincent de Paul mourut à Saint-Lazare en 1660.

3. Voir dans la base Mémoire : image APMH00037791 et image APMH00037792.

4. Beaumont-Maillet, Laure. Vie et histoire du Xe arrondissement. Paris : Hervas, 1988, p. 55.

5. Les sœurs de Marie-Joseph pour les prisons ou Sœurs des Prisons est un ordre fondé à Lyon en 1805 par Mademoiselle Duplaix.

6. Archives de la Seine 4 AZ 1154.

7. Une vue perspective conservée aux archives de l'A.P.-H.P. [HZ 107] permet de bien connaître l'aspect de cet ensemble au milieu du XIX ${ }^{\mathrm{e}}$ siècle.

8. Voir dans la base Mémoire : image IVR11_80941447Z, image IVR11_80941448Z. Voir dans la base Mérimée : notice IA94000305.

9. La prison se trouvait rue du Puits-de-l'Ermite, $V^{\mathrm{e}}$ arr. Voir: Pinon, Pierre. Louis-Pierre et Victor Baltard. Paris : Monum, 2005, p. 51-55.

10. Voir dans la base Mémoire : image APMH00038462, image APMH00038463.

11. Voir dans la base Mémoire : image APLCR00339, image 1FS00817. Voir dans la base Mérimée : notice PA00117980.

12. Pinon, Pierre. Louis-Pierre et Victor Baltard. Paris : Monum, 2005, p. 47.

13. L.-P. Baltard, cité par Foucaud, O. Iconographie de l'architecture : la prison française au XIX ${ }^{\mathrm{e}}$ siècle, dernier avatar du couvent, G.B.A., 136e année, nov. 1994, p. 197.

14. Une entrée depuis l'atelier de grosse couture (bas-côté nord), une depuis le vestibule attenant à la sacristie (bas-côté sud) et l'entrée principale à l'est depuis le préau des condamnées et des prévenues.

15. D'une grande ampleur, la prison circonscrit 4 cours (ill. 2).

16. Voir sur le site de l'agence Roger-Viollet : photographie $n^{\circ}$ 33260-10.

17. Foucaud, O. Iconographie de l'architecture : la prison française au XIX ${ }^{\mathrm{e}}$ siècle, dernier avatar du couvent, G.B.A., 136 année, nov. 1994, p. 202.

18. En 2004, lors de la constitution du dossier de recensement au titre des monuments historiques, un petit autel et divers ex-votos s'y trouvaient encore.

19. Ce décor était visible en 2004, lors du déplacement des dalles du faux plafond qui masquait l'ensemble du voûtement. Voir photographie conservée au musée Carnavalet [146 E Topo].

20. Voir sur le site de l'agence Roger-Viollet : photographie $n^{\circ} 33260-14$.

21. Voir Médiathèque du patrimoine, [cote 0081/075610/008 : courrier relatif à la protection et à la démolition de la prison (1884-1885) et (1931-1933)] et voir procès-verbaux de la commission du Vieux-Paris (juillet 1912, janvier et février 1928, mars et novembre 1930, mai 1931, janv., fév., avril et juin 1933).

22. Procès-verbal de la C.V.P. du 28 janvier 1928.

23. Procès-verbal de la C.V.P. du 25 février 1928. 
24. Procès-verbal de la C.V.P. du 29 mars 1930.

25. Procès-verbal de la C.V.P. du 9 mai 1931.

26. Médiathèque du patrimoine: articles du Matin, de Comedia et du Petit Parisien [cote 0081/075610/008].

27. Archives de la Seine [D7N4 41].

28. Voir dans la base Mérimée : notice PA75100008.

\section{RÉSUMÉS}

Après 1794 l'ancien couvent de la Congrégation de saint Vincent de Paul, transformé en prison pour femmes, était devenu un lieu sinistre et mal entretenu qui menaçait ruine. L'architecte de l'administration pénitentiaire demande alors la démolition d'urgence de l'ancienne église gothique. En 1823, une nouvelle chapelle et une infirmerie sont construites par Louis-Pierre Baltard (1764-1846) nommé architecte des prisons du département de la Seine dix ans auparavant. La chapelle de plan pseudo-basilical avec une abside semi-circulaire est décorée de colonnes doriques. Elle est caractérisée par ses deux niveaux, une sorte de sous-sol ouvert et des galeries latérales de belle ampleur. Cette disposition permettait de différencier l'entrée des détenues de droit commun de celle des prostituées et évitait qu'elles se trouvent ensemble dans la nef. Cette église est un témoin de l'architecture religieuse de la Restauration, période assez peu féconde, et une œuvre bien représentative de Louis-Pierre Baltard qui a beaucoup participé aux débats architecturaux de son temps, mais qui a assez peu construit. L'ancienne infirmerie et la chapelle sont inscrites au titre des monuments historiques depuis le 28 novembre 2005.

After 1794, the former convent of the Congregation of Saint Vincent de Paul, re-used as a prison for women, had become a sinister and badly maintained building which threatened ruin. The architect of the penitentiary administration demanded the urgent demolition of the convent's old Gothic church. In 1823, a new chapel with an infirmary were built to the designs of LouisPierre Baltard (1764-1846), who had been appointed as architect for the prison administration of the Seine department ten years earlier. The chapel had a pseudo-basilical ground plan with a semi-circular apse, and had Doric columns. The building is characterised by its construction on two levels, with a sort of open basement level and generous side galleries. This design was intended to separate the entrances to keep the common law prisoners apart from the prostitutes and to prevent them from mingling in the nave. The church bears witness then to religious architecture during the period of the Restoration (1815-1830), a period which was not particularly prolific in its church building. It is also representative work for the architect LouisPierre Baltard who was an active participant in the architectural debates of his time but who in fact built very little. The former infirmary and the chapel were given statutory protection (inscription) on 28 November 2005. 


\section{INDEX}

Mots-clés : architectonographie des prisons, architecture religieuse de la Restauration, couvent de la Congrégation de saint Vincent de Paul, maison de santé Saint-Lazare, prison Saint-Lazare, chapelle Saint-Lazare, Bicêtre, prison de La Force, prison des Madelonnettes, prison de Draguignan, chapelle de la prison Sainte-Pélagie, palais de justice de Lyon, Congrégation de saint Vincent de Paul, hospitaliers Saint-Lazare de Jérusalem, lazaristes, Sœurs de Marie-Joseph pour les Prisons, Louis-Pierre Baltard, Gaston Lefol, baron Delessert, comte de Chabrol, Mme Caillaux, Louise Michel, Mata Hari

Keywords : religious architecture of the Restoration, convent of the congregation of Saint Vincent de Paul, Saint-Lazare 'maison de santé', Saint-Lazare prison, Saint-Lazare chapel, La Force prison, Madelonnettes prison, Draguignan prison, Chapel of the Sainte-Pélagie prison, Lyon courthouse, Congregation of Saint Vincent de Paul, Saint-Lazare Hospitallers of Jerusalem, lazarists, Marie-Joseph sisters for prisons, architectural iconography of prison

\section{AUTEUR}

\section{AGNÈS CHAUVIN}

Documentaliste-recenseur, Direction régionale des affaires culturelles d'Île-de-France.

agnes.chauvin@culture.gouv.fr 